\title{
Electrosynthetically patterned conducting polymer films for investigation of neural signaling
}

\author{
Daniel T. Simon and Sue A. Carter ${ }^{\mathrm{a})}$ \\ Physics Department, University of California, Santa Cruz, California 95064
}

(Received 10 January 2006; accepted 4 April 2006; published online 26 May 2006)

\begin{abstract}
The ion-mediated conduction and versatility of device fabrication of conducting polymers provide a route to the study of neural signaling. Patterned junctions of conducting polypyrrole have been electropolymerized on commercially available microelectrode arrays, with typical dimensions $200 \mu \mathrm{m}$ between electrodes, each electrode being $30 \mu \mathrm{m}$ in diameter. Tetrabutylammonium perchlorate or sodium $p$-toluenesulfonate were used as electrolyte/counterion in the organic solvent. Individual polypyrrole junctions, when synthesized and connected in a three-electrode configuration, exhibit current-switching behavior analogous to neural weighting. Junctions copolymerized with thiophene exhibit current rectification and the nonlinear current-voltage behavior requisite for complex neural systems (i.e., the activation function). (C) 2006 American Institute of Physics. [DOI: 10.1063/1.2200348]
\end{abstract}

\section{INTRODUCTION}

The tunable conductivity and ease of synthesis of conducting polymer films make them ideal for the development of structures exhibiting "neural" dynamics. Like biological neurons, conduction within these films is mediated by ions. Polymers depend on the proximity of counterions to the $\pi$-conjugated backbone to stabilize the charge carriers (solitons or polarons), ${ }^{1}$ while biological neurons depend on the influx and efflux of ions to produce their electronic signals. Controlling the flow of counterions into and out of these polymer films determines the overall conductivity in a way analogous to weighting of algorithmic neurons (e.g., perceptrons $s^{2,3}$ ). Furthermore, the unique current versus voltage characteristics of certain blends of conducting polymers provide the nonlinear output versus input requisite for the more complicated algorithmic networks which mirror our own biological system. ${ }^{4}$ These junctions thus provide an experimental platform which lies between conventional semiconductor-based neural computing, based on purely electronic conduction, and our own biological neural network, which is based on ionic and chemical signal conduction. Extended networks of such junctions could then elucidate an understanding of emergent dynamics in neural processing that would be unattainable in a purely electronic system.

Recent attention has been paid to the use of polypyrrole (PPy) and polythiophene (PThio) for these purposes. ${ }^{5,6}$ These conducting polymers can be synthesized electrochemically in situ in their conducting state, ${ }^{7}$ and thus provide an accessible route to the fabrication of extended networks. Microelectrode arrays (MEAs) have also recently been employed as the working electrode(s) in the synthesis and characterization of conducting polymer films. ${ }^{8,9}$ These devices, developed for analysis of brain slices and cultured neural networks in vitro,

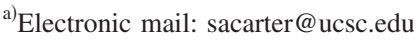

have individually addressable electrodes embedded in an insulating substrate and are thus also ideal for use in microscale electrochemistry. Typical dimensions are $\sim 500 \mu \mathrm{m}^{2}$ electrodes spaced $\sim 200 \mu \mathrm{m}$ apart. The advantage of using these arrays is that polymer electronics can be patterned by selective electropolymerization above individually addressable electrodes. Multiple junctions could then be synthesized in a "connect-the-dots" fashion, yielding patterened conducting polymer networks.

To date, all researches into such neural polymer systems have either been done on a macroscopic scale $(\sim 10 \mathrm{~cm})$ without the use of a potentiostat, ${ }^{6}$ employed potentially over oxidizing voltages in the electrosynthesis or "weighting" stages, ${ }^{10}$ or used polymers synthesized ex situ. ${ }^{5}$ Even previous work involving PPy on MEAs (Ref. 8) relied on extended interdigitated electrodes, not today's standard MEA geometry.

In this article, we report on the electrical dynamics of two- and three-electrode junctions electropolymerized on a commercially available MEA. In the three-electrode case, the PPy junctions each have an input, output, and control electrode, and we investigate the response of the output to voltages applied at the control electrode, i.e., neural weighting of signals. In the two-electrode case, the PPy/Thio blend junctions consist solely of input and output, and we investigate the nonlinear current versus voltage behavior required for complex neural networks (i.e., the activation, or transfer, function relating the summed inputs to the output).

\section{EXPERIMENT}

\section{A. Materials and apparatus}

Pyrrole monomer, thiophene monomer, sodium p-toluenesulfonate (NaTS), and tetrabutylammonium perchlorate $\left(\mathrm{TBAClO}_{4}\right)$ were used as received from SigmaAldrich. Anhydrous acetonitrile (Alfa Aesar) was also used as received. All solutions were prepared in a nitrogen dry 


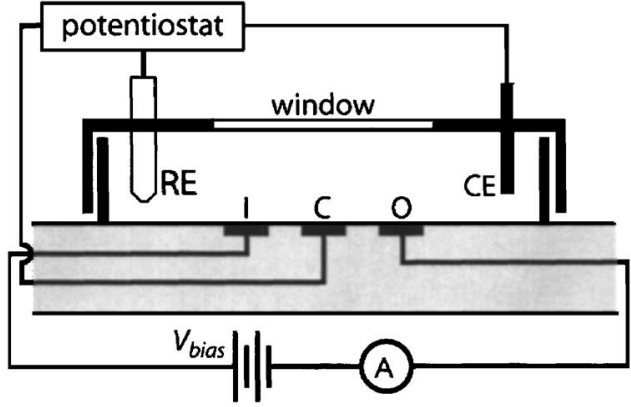

FIG. 1. The two circuits used in three-electrode analysis showing input (I), output $(\mathrm{O})$, and control (C) MEA electrodes, Pt wire counter electrode (CE), and SCE reference electrode (RE). Only the control electrode is part of the three-terminal potentiostat circuit during the analysis. During polymerization, $\mathrm{I}$ and $\mathrm{O}$ are connected to $\mathrm{C}$ as the composite working electrode.

box and stored in the dark at $2{ }^{\circ} \mathrm{C}$ under nitrogen. The MultiChannelSystems MEA (200/30 $\mu \mathrm{m}-\mathrm{TiN} / \mathrm{SiN}$ model) was sonicated for $2 \mathrm{~min}$ in de-ionized water and then rinsed repeatedly with de-ionized water and ethanol before each use.

The electrochemical cell used for electropolymerization and three-electrode analysis consisted of the TiN electrodes of the MEA as working electrode(s), a $0.5 \mathrm{~mm}$ diameter platinum wire (Aldrich) as counter electrode, and a saturated calomel electrode (SCE) (Accumet) as reference (Fig. 1). The reference electrode was inserted into the solution using a $3 \mathrm{ml}$ luggin capillary. All experiments were carried out in air. The cell was controlled via an EG\&G Princeton Applied Research 362 potentiostat with dual Hewlett Packard 3245A universal sources. Data were collected on a Keithley 2000 digital multimeter, and the system was imaged during electrosynthesis using a QImaging QCapture charge-coupled device (CCD) camera mounted on a Technical Instruments K2S-BIO confocal microscope (on a Nikon upright microscope base).

The solutions used for the two-electrode analysis consisted of $0.2 \mathrm{M} \mathrm{TBAClO}_{4}$ in acetonitrile with $0.5 \mathrm{M}$ pyrrole (" $1: 0$ " solution), $0.25 M$ pyrrole with $0.25 M$ thiophene (" $1: 1$ " solution), and $0.167 M$ pyrrole with $0.333 M$ thiophene ("1:2" solution).

\section{B. Electropolymerization}

For the three-electrode devices, PPy was electropolymerized into its conducting state using a solution of $0.5 \mathrm{M}$ pyrrole monomer and $0.2 \mathrm{M}$ counterion salt $\left(\mathrm{NaTS}\right.$ or $\left.\mathrm{TBAClO}_{4}\right)$ in acetonitrile. Once added to the well on the MEA (Fig. 1), the solution was capped to prevent evaporation. Following the work of Diaz et al., ${ }^{7}$ a voltage of $0.8 \mathrm{~V}$ vs SCE was applied until the desired area of PPy was formed ( $\sim 30-60 \mathrm{~min}$ for $\left.\sim 0.2 \mathrm{~cm}^{2}\right)$. Polymerization was carried out on three adjacent MEA electrodes, labeled I, O, and C in this article for input, output, and control. Voltage was supplied to all three electrodes until just before the polymer films above each electrode were large enough to make electrical contact with the adjacent films. At this point, the outer two electrodes (I and O) were disconnected from the voltage supply, leaving only the central electrode (C) as part of the electrochemical circuit. Current was supplied through the central electrode until its polymer film made electrical con-

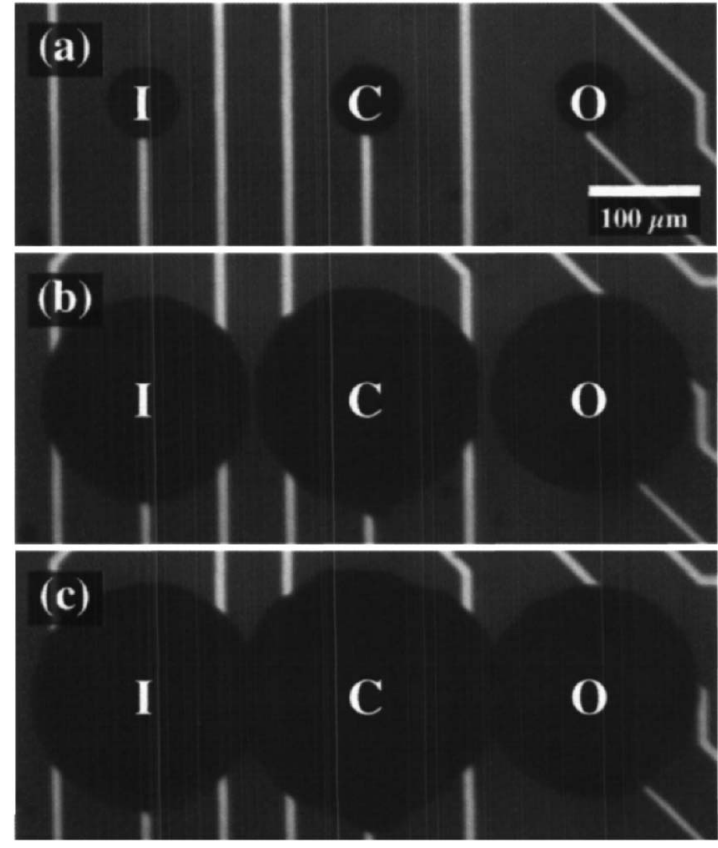

FIG. 2. Polymerization above three MEA electrodes at three times (where I, $\mathrm{O}$, and $\mathrm{C}$ stand for input, output, and control: (a) at 29 min with all three electrodes connected as the working electrode; (b) at $73 \mathrm{~min}$, when the I and $\mathrm{O}$ electrodes have been disconnected from the voltage supply; and (c) at $80 \mathrm{~min}$, after the $\mathrm{C}$ film has made electrical contact with the I and $\mathrm{O}$ films.

tact with the outer two films (Fig. 2). The current versus time plot shows that the three electrodes form a contiguous conducting film (Fig. 3). The steady increase in current in Fig. 3 is indicative of the increase in surface area of the PPy films: as more surface area is created, more sites are made available to add monomer units. The two-electrode PPy/Thio blend devices were electropolymerized in a similar fashion using a solution of pyrrole and thiophene in $0.2 \mathrm{M} \mathrm{TBAClO}_{4}$ acetonitrile (1:0, 1:1, or $1: 2$, as described above). In this case, instead of completing the polymerization using the $\mathrm{C}$ electrode, the $\mathrm{O}$ electrode was used to complete the polymer film in the direction of the I electrode.

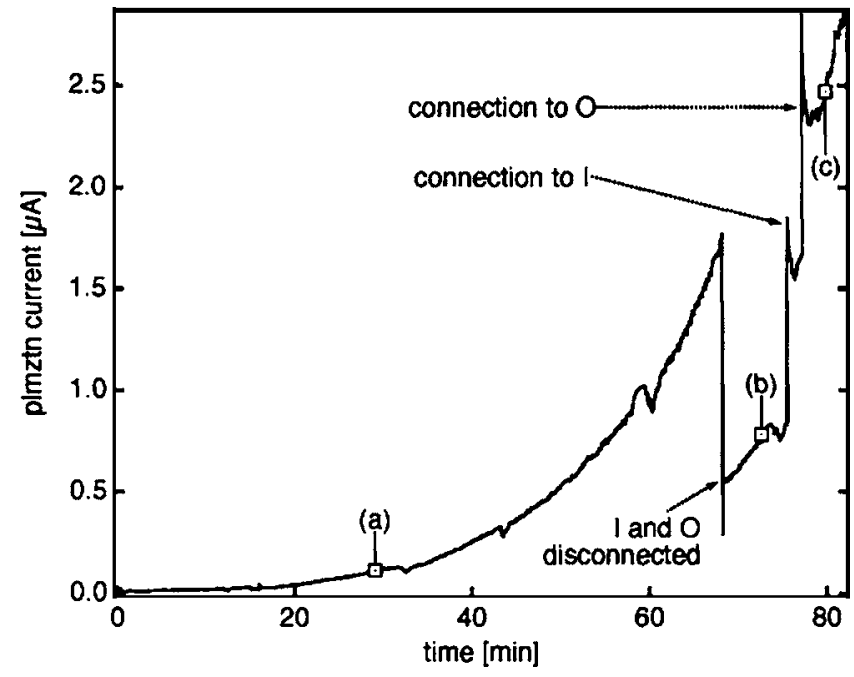

FIG. 3. Cell current vs time plot associated with the polymerization of Fig. 2. The $\mathrm{I}$ and $\mathrm{O}$ electrodes are disconnected at $68 \mathrm{~min}$, making the $\mathrm{C}$ the only working electrode. At $75 \mathrm{~min}$, the $\mathrm{C}$ film makes electrical contact with the I film, and then at $77 \mathrm{~min}$, with the $\mathrm{O}$ film. The tags labeled a, b, and c, correspond to the images of Fig. 2. 


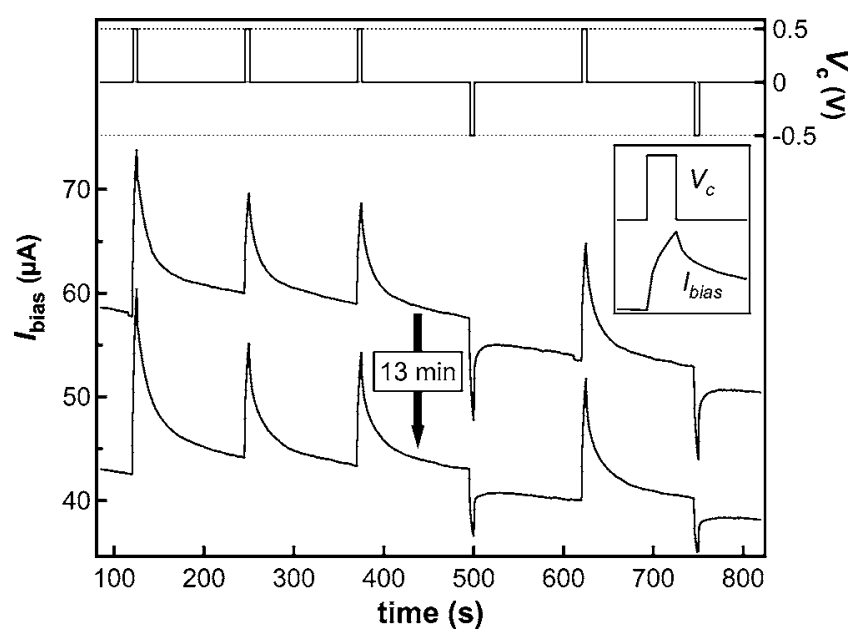

FIG. 4. $I_{\text {bias }}$ (raw data) vs time in response to $V_{c}= \pm 0.5 \mathrm{~V}$ (vs SCE) pulses of $5 \mathrm{~s}$ duration, spaced $120 \mathrm{~s}$ apart. The electrolyte solution was $0.2 \mathrm{M}$ $\mathrm{TBAClO}_{4}$. The plot cuts off the first $100 \mathrm{~s}$ during which the system was left to equilibrate. The inset shows detail of $I_{\text {bias }}$ rise and fall for a positive $V_{c}$ pulse (5 s in duration).

\section{Device Analysis}

The three-electrode analysis was performed by "ionic gating" of the polymer film via voltages applied to the C electrode. The input-output circuit and control circuit are shown in Fig. 1. A constant voltage, $V_{\text {bias }}$, was supplied across the I-O junction and the I-O current, $I_{\text {bias }}$, was recorded. Potentiostat-supplied voltage pulses (versus SCE), $V_{c}$, were then supplied through the control electrode to modulate $I_{\text {bias. }}$. Junction rectification analysis was performed using the two-electrode junction with single input and output. An increasing ramp voltage (Fig. 8 inset) was applied between the two electrodes (i.e., through the polymer bulk), and the current was measured as a function of the bias voltage. To prevent continued polymerization during analysis, the electrochemical cell was rinsed and refilled with a solution containing only the counterion $\left(\mathrm{NaTS}\right.$ or $\left.\mathrm{TBAClO}_{4}\right)$ in acetonitrile.

\section{RESULTS AND DISCUSSION}

\section{A. Three-electrode ionic gating analysis}

The results of a typical three-electrode experiment are shown in Fig. 4, i.e., the conductivity of the input-output channel can be quasipermanently modulated (weighted) by $V_{c}$. This behavior is explained by means of the polaron/ bipolaron picture of conduction. ${ }^{1,11}$ When $V_{c}>0$ (vs SCE), PPy is oxidized and negative counterions $\left(\mathrm{TS}^{-}\right.$or $\mathrm{ClO}_{4}^{-}$) are attracted toward the film above the $\mathrm{C}$ electrode. PPy is known to be in its conducting state when oxidized, ${ }^{7}$ i.e., positive (bi)polarons are the charge carriers. When negative counterions are brought into proximity with the $\pi$-conjugated backbone of the polymer, positive (bi)polarons can readily be stabilized (charge neutralized) over large interchain distances. In this manner, the conductivity of the overall film increases as hopping sites are made available. Likewise, for $V_{c}<0$ (vs SCE), PPy is reduced and the nega-

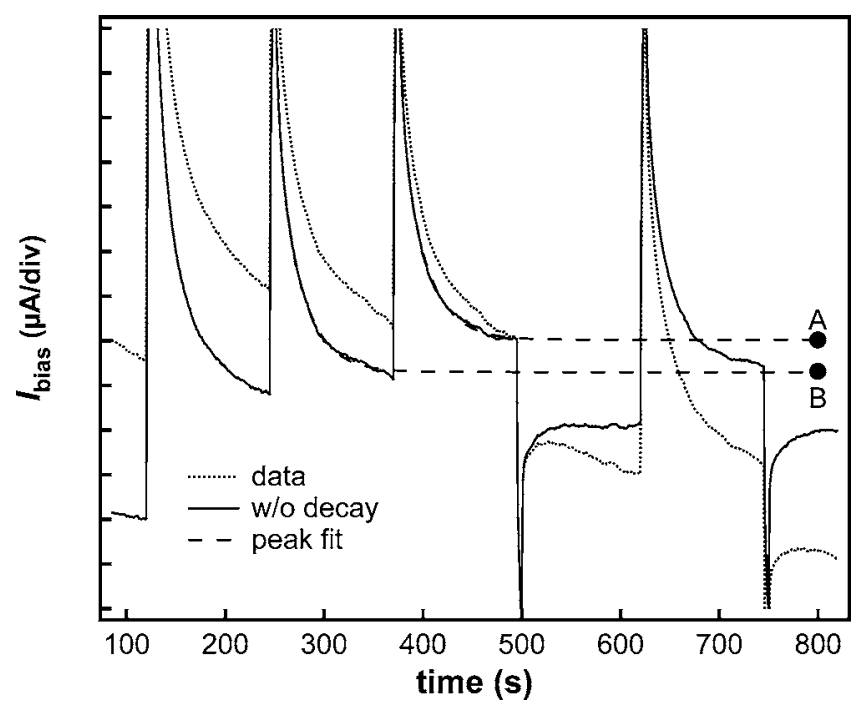

FIG. 5. Data with overall decay subtracted and double-exponential fits to peaks 2 and 3. The two dashed lines illustrate the calculation of $\Delta I$ : point $\mathrm{A}$ is $I_{\mathrm{after}}^{800}$, and point $\mathrm{B}$ is $I_{\text {before }}^{800}$, so that $\Delta I=I(\mathrm{~A})-I(\mathrm{~B}) / I(\mathrm{~B})$ for peak 3 . The scale on $I_{\text {bias }}$ is $1 \mu \mathrm{A} /$ division.

tive counterions requisite for enhanced conduction are swept away from the polymer film, thus decreasing the conductivity.

The overall decrease in $I_{\text {bias }}$ with time $\left(\tau_{\text {global }} \approx 45 \mathrm{~min}\right)$ is due to the exposure of the system to oxygen trapped under the cell cap (Fig. 1) and the evaporation of acetonitrile from the electrolyte solution. Both result in irreversible oxidation of the PPy. In the case of evaporation, the loss of solvent results in an increased salt concentration, leading to irreversible oxidation of the PPy by covalent bonding of the counterions. The presence of oxygen (i.e., free $\mathrm{OH}^{-}$) can also cause irreversible covalent bonding. ${ }^{12}$

With each positive $V_{c}$ pulse ( $5 \mathrm{~s}$ duration), $I_{\text {bias }}$ rises during the pulse, then relaxes to a current greater than before the pulse (vice versa for negative $V_{c}$ pulses). Examining the lower of the two traces in Fig. 4, we first divide out the overall decay using an exponential fit ( $\tau=4984 \mathrm{~s})$ to the data past $800 \mathrm{~s}$, most of which is not shown in the figure, to account for the steady oxidation referred to above. Each peak was then fit with a double exponential,

$$
f(t)=A+B e^{-\left(t-t_{0}\right) / \tau_{1}}+C e^{-\left(t-t_{0}\right) / \tau_{2}} .
$$

The motivation for this function arises from the two ion diffusive processes at work: the counterions at the polymer/ solution interface quickly migrate away ( $\tau_{1}$, fast), while the counterions within the polymer bulk must make their way to this interface $\left(\tau_{2}\right.$, slow) before migrating away along the concentration gradient in solution. The change in current $\Delta I$ was calculated by comparing these fits extrapolated out to $800 \mathrm{~s}$, an arbitrary time chosen after the last $V_{c}$ pulse (Fig. 5 and Table I):

$$
\Delta I=\left[I_{\text {after }}^{800}-I_{\text {before }}^{800}\right] / I_{\text {before }}^{800},
$$

where $I_{\text {before }}^{800}$ is the current at $800 \mathrm{~s}$ extrapolated from the fit before the $V_{c}$ pulse, and $I_{\text {after }}^{800}$ is the current at $800 \mathrm{~s}$ extrapolated from the fit after the $V_{c}$ pulse (Fig. 5). 
TABLE I. Extrapolated percent changes in $I_{\text {bias }}$ from Fig. 5. The peaks are numbered with increasing time. $B / C$ is in reference to the fit parameters of Eq. (1); it is the ratio of carriers associated with fast decay $B$ and slow decay C.

\begin{tabular}{ccccc}
\hline \hline Peak & $\begin{array}{c}\tau_{1}{ }^{\mathrm{a}} \\
(\mathrm{s})\end{array}$ & $\begin{array}{l}\tau_{2}{ }^{\mathrm{b}} \\
(\mathrm{s})\end{array}$ & $B / C^{\mathrm{c}}$ & $\begin{array}{l}\Delta I^{\mathrm{d}} \\
(\%)\end{array}$ \\
\hline 1 & 11.3 & 49.0 & 1.76 & +7.0 \\
2 & 3.2 & 24.0 & 0.42 & +2.1 \\
3 & 3.7 & 24.8 & 0.45 & +1.7 \\
4 & 0.6 & 13.5 & 0.44 & -0.4 \\
5 & 3.0 & 23.0 & 0.42 & +3.3 \\
6 & 1.1 & 17.1 & 0.45 & -3.6 \\
\hline \hline
\end{tabular}

average error \pm 0.2 .

${ }^{\mathrm{b}}$ Average error \pm 0.8 .

${ }^{\mathrm{c}}$ Average error \pm 0.04 .

${ }^{\mathrm{d}}$ Average error (for peaks 2-6) \pm 0.05 .

The results of the $\Delta I$ calculations (Table I) show that $V_{c}=+0.8 \mathrm{~V}$ (vs SCE) pulses increase $I_{\text {bias }}$ by $\sim 2 \%-7 \%$, and $V_{c}=-0.8 \mathrm{~V}$ (vs SCE) pulses decrease $I_{\text {bias }}$ by $\sim 4 \%$. Peak 1 is unique in both $\Delta I$ and its $\tau_{i}^{\prime}$ 's since it is the response to the first $V_{c}$ pulse after 2 min of equilibration. Peaks 2 and 3 are qualitatively similar and peak 5 stands out in its $\Delta I$ because it was preceded by a conductivity reducing negative $V_{c}$ pulse. The two negative peaks (4 and 6) are also qualitatively similar, with peak 4 showing a larger $\Delta I$ since it followed three positive $V_{c}$ pulses rather than one. With each successive $V_{c}$ pulse, the polymer is nearing a saturation of counterions. Thus, a limiting of response with successive positive pulses is observed: $\Delta I_{\text {peak } 1}>\Delta I_{\text {peak } 2}>\Delta I_{\text {peak3 }}$.

The above data were for a $0.2 M \mathrm{TBAClO}_{4}$ solution. The response is quantitatively different in $0.2 M$ NaTS electrolyte (Fig. 6). Most notably, the $\tau_{i}$ 's are shorter due to the difference in size between the $\mathrm{TS}^{-}$and $\mathrm{ClO}_{4}^{-}$anions (Fig. 7). The increased $\Delta I$ is explained both by the higher mobility of the smaller anions and the increased strength of the gating pulses $\left(V_{c}=2 \mathrm{~V}\right.$ vs $\left.\mathrm{SCE}\right)$ : more anions are incorporated into the polymer film with each gating pulse. The decrease in $\Delta I$ with successive $V_{c}$ pulses is again observed.

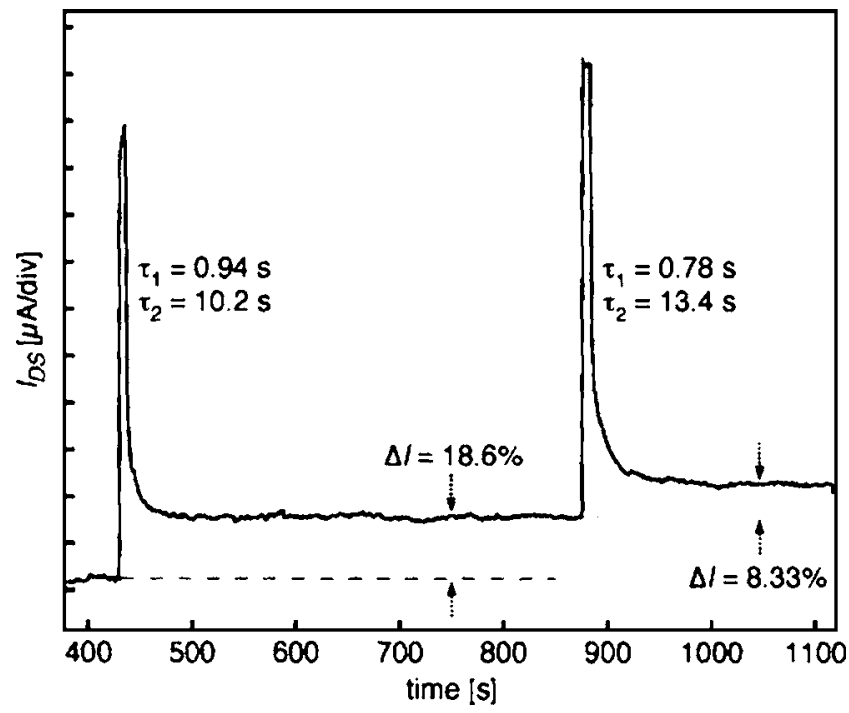

FIG. 6. $I_{\text {bias }}$ vs time in response to $V_{c}=2 \mathrm{~V}$ (vs SCE) pulses of $5 \mathrm{~s}$ duration in $0.2 M$ NaTS electrolyte solution. An exponential decay $(\tau=3046 \mathrm{~s})$ has been subtracted from the data.

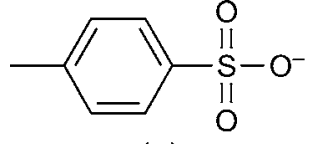

(a)<smiles>[O-][Cl+3]([O-])([O-])[O-]</smiles>

(b)
FIG. 7. Formulas of (a) $p$-toluenesulfonate $\left(\mathrm{TS}^{-}\right)$and (b) perchlorate $\left(\mathrm{ClO}_{4}^{-}\right)$ anions. Note that the $\mathrm{TS}^{-}$is larger by an entire phenyl group.

\section{B. Two-electrode junction analysis}

Results for the two-electrode analysis are shown in Fig. 8. As the ratio of thiophene in the polymerization solution increases, rectification of the junction becomes evident. Unlike previous experiments on PPy/Thio rectification, ${ }^{13}$ this effect cannot be attributed to electrochemical changes in the polymer brought about by simple copolymerization of the pyrrole and thiophene. Since these junctions were polymerized at $1 \mathrm{~V}$ vs SCE, which is above the oxidation potential of pyrrole $(\sim 0.7 \mathrm{~V})$ but below the oxidation potential of thiophene $(\sim 1.7 \mathrm{~V}),{ }^{14}$ the resulting polymer must be almost entirely composed of pyrrole. ${ }^{15}$ At this voltage, a small amount of thiophene is polymerized, ${ }^{16}$ but the rate is much slower than for the pyrrole. The junctions are then PPy films with embedded thiophene oligomers. During the polymerization, these oligomers are subjected to the electric field within the PPy bulk, which is roughly oriented radially outward from the MEA electrode toward the perimeter of the film (i.e., toward the polymerization sites). Since the junction is completed by polymerizing the film above the $\mathrm{O}$ electrode until it reaches the I film, the electric field present on the completion of the junction points from the $\mathrm{O}$ to the $\mathrm{I}$ electrodes. The polarizability of oligothiophenes is well known, ${ }^{17,18}$ but in this case, the final electric field "freezes" the polarization of the oligomers caused by trapped charge in the form of (bi)polarons, as discussed above. These polarized oligomers remain aligned with the final polymerization field and either enhance (forward bias) or partially cancel (reverse bias) the fields applied to the junction during analysis. The

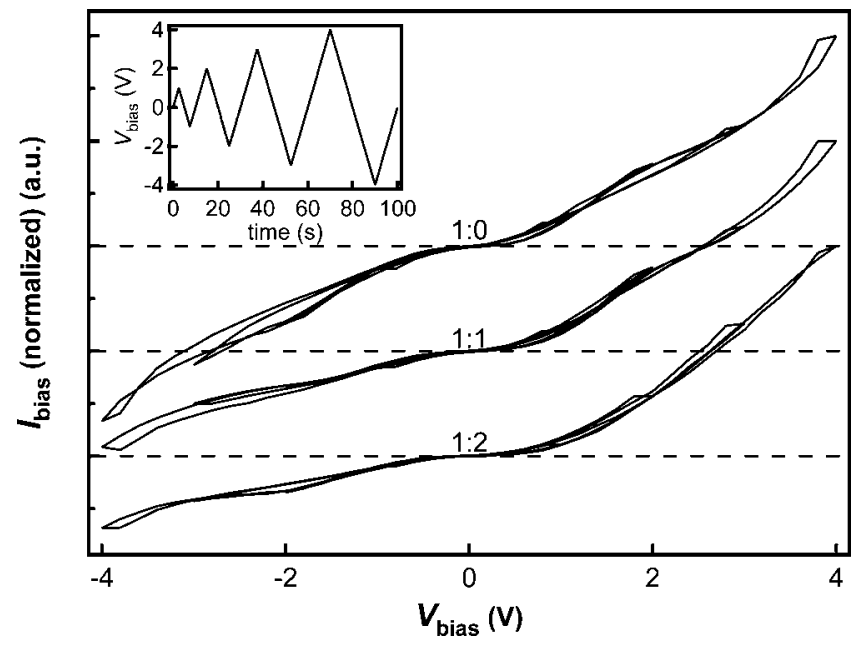

FIG. 8. Typical current vs voltage for PPy and PPy/Thio blend twoelectrode junctions. The current is normalized to the maximum positive current. The labels indicate the pyrrole:thiophene ratio used in the electropolymerization of the junctions. The inset shows the increasing ramp $(400 \mathrm{mV} / \mathrm{s}) V_{\text {bias }}$ vs time. 
TABLE II. Quantitative analysis of rectification data in Fig. 8. Current in forward bias, $I_{+}$, is compared to current in reverse bias, $I_{-}$, at \pm 2 and $\pm 4 \mathrm{~V}$. The larger the ratio, the more pronounced the rectification.

\begin{tabular}{ccc}
\hline \hline Film & $I_{+} / I_{-}( \pm 2 \mathrm{~V})$ & $I_{+} / I_{-}( \pm 4 \mathrm{~V})$ \\
\hline $1: 0$ & 1.2 & 1.2 \\
$1: 1$ & 1.8 & 2.2 \\
$1: 2$ & 2.2 & 2.9 \\
\hline \hline
\end{tabular}

quantitative results of this rectification are shown in Table II. The slight rectification observed in the 1:0 film is attributed to a small number of trapped pyrrole oligomers.

\section{CONCLUSION}

Simple conducting polymer junctions, as reported in this article, can be utilized for the modeling and analysis of neural systems. We have demonstrated that microscale junctions are easily patterned using commercially available microelectrode arrays and that larger networks could thus be synthesized in situ in an intricate connect-the-dots fashion. We have also shown that by means of an integrated control electrode, these junctions can be electrochemically weighted. In an extended network of such junctions, this individual weighting could lead to system memory. Finally, we have provided evidence of the nonlinear (in this case, rectifying) current versus voltage characteristics required for these junctions to function as elements in complicated neural circuits. The simple two- and three-electrode junctions demonstrated here are a first step toward the fabrication of larger networks of many such junctions. The essential qualities of patternability, signal weighting, and nonlinearity, indicate that such networks would mimic the basic features of algorithmic-or even biological-neural networks, and elucidate the dynamics of these systems which emerge as the number of individual elements increases.

\section{ACKNOWLEDGMENT}

This research was funded by a grant from the National Science Foundation (Electrical and Communication Systems Grant No. 0101794).

${ }^{1}$ A. J. Heeger, S. Kivelson, and J. Schrieffer, Rev. Mod. Phys. 60, 781 (1988), http://dx.doi.org/10.1103/RevModPhys.60.781

${ }^{2}$ F. Rosenblatt, Psychol. Rev. 62, 386 (1958).

${ }^{3}$ D. E. Rumelhart, G. E. Hinton, and R. J. Williams, Parallel Distributed Processing (MIT, Cambridge, MA, 1986), Vol. 1, Chap. 8, pp. 318-362, http://cognet.mit.edu/library/books/view?isbn $=026268053 \mathrm{X}$

${ }^{4}$ D. E. Rumelhart, G. E. Hinton, and J. L. McClelland, Parallel Distributed Processing (MIT, Cambridge, MA, 1986), Vol. 1, Chap. 2, pp. 4576, http://cognet.mit.edu/library/books/view?isbn $=026268053 \mathrm{X}$

${ }^{5}$ K. Yoshino, T. Kuwabara, and T. Kawai, Jpn. J. Appl. Phys., Part 2 29, L995 (1990), http://jjap.ipap.jp/link?JJAP/29/L995/

${ }^{6}$ M. Fujii, T. Takahashi, H. Ihori, K. Arii, and K. Yoshino, Synth. Met. 101, $397 \quad$ (1999), http://dx.doi.org/10.1016/S03796779\%2898\%2900990-4

${ }^{7}$ A. F. Diaz, J. I. Castillo, J. A. Logan, and W. Y. Lee, J. Electroanal. Chem. $\quad 129, \quad 115 \quad$ (1981), http://dx.doi.org/10.1016/03681874(81)87387-X

${ }^{8}$ G. P. Kittlesen, H. S. White, and M. S. Wrighton, J. Am. Chem. Soc. 106, 7389 (1984)

${ }^{9}$ X. Y. Cui and D. C. Martin, Sens. Actuators B 89, 29 (2003), http:// dx.doi.org/10.1016/S0925-4005\%2802\%2900448-3

${ }^{10}$ T. Kawai, H. Motobayashi, T. Kuwabara, and K. Yoshino, Jpn. J. Appl. Phys., Part 2 30, L622 (1991), http://jjap.ipap.jp/link?JJAP/30/L622/

${ }^{11}$ W. P. Su, J. R. Schrieffer, and A. J. Heeger, Phys. Rev. B 22, 2099 (1980), http://dx.doi.org/10.1103/PhysRevB.22.2099

${ }^{12}$ F. Beck, P. Braun, and M. Oberst, Ber. Bunsenges. Phys. Chem. 91, 967 (1987).

${ }^{13}$ M. Fujii, N. Takahashi, H. Ohnishi, and K. Yoshino, Synth. Met. 85, 1409 (1997), http://dx.doi.org/10.1016/S0379-6779\%2897\%2980296-2

${ }^{14}$ H. Siegerman, Techniques of Chemistry, (Wiley, New York, 1975), Vol. V, p. 667.

${ }^{15}$ S. Kuwabata, S. Ito, and H. Yoneyama, J. Electrochem. Soc. 135, 1691 (1988).

${ }^{16}$ G. Tourillon and F. Garnier, J. Electroanal. Chem. 135, 173 (1982), http://dx.doi.org/10.1016/0022-0728(82)90015-8

${ }^{17}$ M.-T. Zhao, B. P. Singh, and P. N. Prasad, J. Chem. Phys. 89, 5535 (1988), http://dx.doi.org/10.1063/1.455560

${ }^{18}$ B. Champagne, D. H. Mosley, and J.-M. André, J. Chem. Phys. 100, 2034 (1994), http://dx.doi.org/10.1063/1.466555 